TOKYO J. MATH.

VOL. 11, No. 2, 1988

\title{
Classification of Reducible Plane Curves
}

\author{
Shigeru IITAKA
}

Gakushuin University

§1. In this paper, we shall study reducible plane curves with two irreducible components from the viewpoint of birational geometry of the projective plane. For instance, two plane curves $C$ and $C^{\prime}$ are said to be birationally equivalent if there exists a birational map $\psi$ of the projective plane such that the proper transform of $C$ coincides with $C^{\prime}$. The proper transform of $C$ by $\psi$ is denoted by $\psi[C]$ and a birational map of the plane into itself is called a Cremona transformation.

In general, we consider reducible curves $D$ on a projective non-singular rational surface. When studying plane curves $C$, we take a birational map from $P^{2}$ onto a rational surface $X$. By taking a suitable birational map, we may assume that $X$ is a non-singular rational complete surface and the reducible curve is a disjoint sum of non-singular curves $D_{1}$ and $D_{2}$ on $X$.

Let $D=D_{1}+D_{2}$ and define $\kappa[D]$ to be the logarithmic Kodaira dimension of the open algebraic surface $X-D$, that is $\bar{\kappa}(X-D)=\kappa\left(K_{X}+D, X\right)$ by definition, where $K_{X}$ is a canonical divisor on $X$. In [5], it was shown that if $\kappa[D]=-\infty$, then $D$ is an exceptional curve of the second kind, in other words, there exists a birational map $\varphi: X \rightarrow X_{1}$ such that $\varphi_{1}(D \cap$ $\operatorname{dom}(\varphi)$ ) is a non-singular point on $X_{1}$, where $\operatorname{dom}(\varphi)$ is the set of points at which $\varphi$ is regular and $\left(\varphi_{1}, \operatorname{dom}(\phi)\right)$ is a representative of $\varphi$. In this case, there exists a birational map $\psi: X \rightarrow \boldsymbol{P}^{2}$ such that the proper transform of $D$ is a sum of two lines.

The purpose of this paper is to study plane curves $D$ satisfying $\kappa[D] \geqq 0$.

Recall that $\kappa[D]$ is a birational invariant. Precisely speaking, two pairs $(B, Y)$ and $(D, X)$ are said to be birationally equivalent, if there exists a birational map $h: X \rightarrow Y$ such that all irreducible components of $D$ correspond birationally to those of $B$ by $h$. If $B$ and $D$ are disjoint unions of non-singular curves, then the spaces of logarithmic $m$-ple 2- 
forms are isomorphic to each other. The dimensions of $H^{\circ}\left(X, \mathcal{O}\left(m\left(K_{x}+\right.\right.\right.$ $D))$ ) are birational invariants for any $m>0$, denoted by $P_{m}[D]$. Recall that $\kappa[D]$ is the degree of $P_{m}[D]$ as a function in $m$, which is therefore the birational invariant of the pair $(D, X)$.

In general, for any curve $C$ on a surface $X$ we can define the $m$ genus $P_{m}[C]$ to be $P_{m}[D]$ and the Kodaira dimension $\kappa[C]$ to be $\kappa[D]$ where $(D, Y)$ is a non-singular pair birationally equivalent to $(C, X)$ (see $[3,5])$.

Main results are summarized as follows:

Suppose that $D$ is a reducible curve with two components on a rational surface $X$.

1) If $\kappa[D] \geqq 0$, then $P_{2}[D]>0$.

2) Pairs $(D, X)$ with $\kappa[D]=0$ or $=1$ are completely classified (see Propositions 3 and 5).

For example, if $D$ consists of two rational curves and if $\kappa[D]=0$, then $(D, X)$ is derived as follows:

Take a sextic curve with two connected components $C_{1}$ and $C_{2}$. Suppose that each $C_{i}$ is a rational curve and that the sum $C_{1}+C_{2}$ has only double points. Then the pair $(D, X)$ derived from the reducible curve $C_{1}+C_{2}$ satisfies that $\kappa[D]=0$. Let $a=\operatorname{deg} C_{1}$ and $b=\operatorname{deg} C_{2}$. Then $(a, b)$ is one of the following pairs of integers: $(1,5),(2,4)$ and $(3,3)$. The pair $\left(C, P^{2}\right)$ of degree $(3,3)$ can be transformed into a pair of degree $(2,4)$ by a Cremona transformation with center $(P, Q, R)$ such that $P$ is the singular point of $C_{1}$ and $Q, R$ are intersection points of $C_{1}$ and $C_{2}$. By a similar Cremona transformation, the pair $(C, X)$ of degree $(2,4)$ is transformed into a pair of degree $(1,5)$.

As a corollary to the result 1 ), we have the following criterion of union of two lines on a projective plane which is an analog of Castelnuovo's criterion of rational surfaces.

THEOREM 1. Let $C$ be a curve with two irreducible components on a projective plane. Then $C$ is transformed into a union of two lines by a Cremona transformation if and only if $P_{2}[C]=0$.

In the case of complete surfaces we have the following result:

Surfaces of Kodaira dimension 2 satisfy $P_{2}>1$.

A similar result is proved for any irreducible plane curves; i.e. for any irreducible curve $C$, we have $P_{2}[C]>1$ if $\kappa[C]=2$ (see Lemma 7 in [5]). But we have a reducible curve $C_{1}+C_{2}$ such that $\kappa\left[C_{1}+C_{2}\right]=2$ and $P_{2}\left[C_{1}+C_{2}\right]=1$.

Note that the same criterion for union of three lines do not hold anymore. 
REMARK. Let $f: V \rightarrow B$ be an elliptic rational surface with one triple fiber. Suppose there exists a singular fiber $F$ with three irreducible components $C_{1}, C_{2}, C_{3}$ meeting at a point $p$ and that

$$
3 K_{V}+C \sim 0 \text { for } C=C_{1}+C_{2}+C_{3} .
$$

Here, $\sim$ denotes the linear equivalence between divisors. Blowing up $V$ at the center $p$, we have a birational morphism $\mu: X \rightarrow V$. Let $D$ be the proper transform of $C$. Then

$$
3 K_{X}+D \sim 3\left(\mu^{*} K_{V}+E\right)+\mu^{*} C-3 E \sim 0 .
$$

Thus $3 K_{X}+D \sim 0$. Hence, $P_{2}[D]=0$ and $P_{3}[D]=1$.

But the author cannot give a concrete example of such an elliptic rational surface.

REMARK. Kawamata informed the author that reducible curves $D$ on a rational surface are exceptional curves of the second kind if and only if $\kappa[D]=-\infty$.

Kawamata's proof depends on the deep analysis of open surfaces developed by Kawamata and Tsunoda, which is not published.

QUESTION. In the above case, does the condition $P_{12}[D]=0$ imply $\kappa[D]=-\infty$ ?

The author would like to thank the referee for his careful reading and valuable comments.

§. We use the notation used in [4] and [5]. Letting $D$ be a disjoint sum of two non-singular irreducible curves $D_{1}$ and $D_{2}$ on $X$, i.e. $D=D_{1}+D_{2}$, we say the pair $(D, X)$ is relatively minimal, if each $D_{i}$ is not an exceptional curve of the first kind and $D \cdot E \geqq 2$ for any exceptional curve of the first kind $E$ on $X$. For simplicity, in what follows, by an exceptional curve we mean an exceptional curve of the first kind.

We fix a relatively minimal pair $(D, X)$ such that $\kappa[D] \geqq 0$. One of the most important problem in birational geometry is to find a good minimal model of objects. Our object here is a birational pair $(D, X)$; thus, we have to find the Zariski decomposition of $K_{X}+D$.

In general, for a $Q$-divisor $\Delta$ on $X$ with $\kappa(\Delta, X) \geqq 0$, we have the following $Q$-divisors $\Delta^{(+)}$and $\Delta^{(-)}$such that

(0) $\Delta=\Delta^{(+)}+\Delta^{(-)}$,

(i) $\Delta^{(+)}$is a nef $\boldsymbol{Q}$-divisor with $\kappa\left(\Delta^{(+)}, X\right) \geqq 0$,

(ii) $\Delta^{(-)}$is an effective $Q$-divisor whose support is a divisor with negative-definite intersection matrix or just 0 , 
(iii) $\Delta^{(+)} \cdot \Delta^{(-)}=0$.

The decomposition is unique and $\Delta^{(+)}$is called the nef part of $\Delta$. For any $m>0, H^{\circ}(X, \mathcal{O}(\operatorname{INT}(m \Delta)))=H^{\circ}\left(X, \mathcal{O}\left(\operatorname{INT}\left(m \Delta^{(+)}\right)\right)\right)$, where the symbol INT( ) denotes the integral part of the $Q$-divisor. By definition, we have $\kappa(\Delta, X)=\kappa\left(\Delta^{(+)}, X\right)$.

§3. First we consider the case in which both $D_{1}$ and $D_{2}$ are rational curves. Then since $\left(K_{X}+D_{1}+D_{2}\right) \cdot D_{i}=-2$ for $i=1$ and 2 , the selfintersection numbers of the $D_{i}$ are negative, denoted by $-\beta_{i}$. If we let $Z=K_{X}+\left(1-2 / \beta_{1}\right) D_{1}+\left(1-2 / \beta_{2}\right) D_{2}$, the nef part of $K_{X}+D$ coincides with that of $Z$ by a property of Zariski decomposition.

Assume $\beta_{1} \leqq \beta_{2}$. If $\beta_{1}=2$, then $Z$ turns out to be $K_{x}+\left(1-2 / \beta_{2}\right) D_{2}$ and the nef part is derived from the relatively minimal model of the pair $\left(D_{2}, X\right)$. Actually, contracting successively exceptional curves $E$ on $X$ such that $E \cdot D_{2} \leqq 1$, we have a birational morphism $\lambda: X \rightarrow Y$ where the image $D^{\prime}$ of $D_{2}$ and $Y$ form a relatively minimal pair $\left(D^{\prime}, Y\right)$.

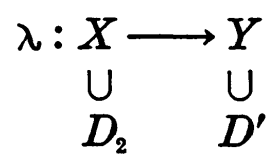

We recall the following result ([5]).

LEMMA 1. Let $D$ be a non-singular rational curve on a non-singular rational surface $X$. Suppose that $(D, X)$ is relatively minimal and $\kappa[D] \geqq 0$. Then $\beta=-D^{2} \geqq 4$ and $Z=K_{x}+(1-2 / \beta) D$ is a nef divisor.

Letting $\beta^{\prime}$ denote $-\left(D^{\prime}\right)^{2}$, we have a nef divisor $K_{Y}+\left(1-2 / \beta^{\prime}\right) D^{\prime}$, which is indicated by $Z^{\prime}$.

Proposition 1. $\lambda^{*}\left(Z^{\prime}\right)$ is the nef part of $Z$, whenever $\beta_{1}=2$.

Proof. Let $F=Z-\lambda^{*}\left(Z^{\prime}\right)$. Then $F$ is effective and is exceptional with respect to $\lambda$ and hence, $F \cdot \lambda^{*}\left(Z^{\prime}\right)=0$. By a property of Zariski decomposition, $Z=\lambda^{*}\left(Z^{\prime}\right)+F$ is the Zariski decomposition.

In the case where $\beta_{1} \geqq 3$, we suppose that $Z$ is not nef. Then there exists an irreducible curve $\Gamma$ such that $Z \cdot \Gamma<0$. Then $\Gamma^{2}<0$. Note that $\Gamma$ is neither $D_{1}$ nor $D_{2}$. This is obvious, since $Z \cdot D_{i}=0$ by definition. Hence,

$$
\Gamma \cdot K_{X}<-\left(1-2 / \beta_{1}\right)\left(\Gamma \cdot D_{1}\right)-\left(1-2 / \beta_{2}\right)\left(\Gamma \cdot D_{2}\right) \leqq 0 .
$$

Therefore, $\Gamma$ is an exceptional curve and 


$$
1>\left(1-2 / \beta_{1}\right) \xi_{1}+\left(1-2 / \beta_{2}\right) \xi_{2},
$$

where $\xi_{i}=D_{i} \cdot \Gamma$ for $i=1,2$. Hence we have the following two cases:

i) $\xi_{1}=2, \xi_{2}=0$. In this case, we have $\beta_{1}=3$ and can derive a contradiction by a similar argument to the proof of Lemma 2 in [5].

ii) $\xi_{1}=1, \xi_{2}=1$. Then $2 / \beta_{1}+2 / \beta_{2}>1$. This case is divided into the following three subcases;

a) $\beta_{1}=3$ and $\beta_{2}=3$,

b) $\beta_{1}=3$ and $\beta_{2}=4$,

c) $\beta_{1}=3$ and $\beta_{2}=5$.

In each subcase, the divisor $D_{1}+D_{2}+\Gamma$ has a negative definite selfintersection matrix. Hence, there exist three non-negative rational numbers $x, y, z$ such that a $Q$-divisor $W=Z-x \cdot D_{1}-y \cdot D_{2}-z \cdot \Gamma$ satisfies that $W \cdot D_{1}=0, W \cdot D_{2}=0$ and $W \cdot \Gamma=0$. Then

$$
z=\left(6-\beta_{2}\right) /\left(2 \beta_{2}-3\right), x=z / 3 \text { and } y=z / \beta_{2} .
$$

Since the nef part of $W$ coincides with that of $Z$, it follows that

$$
\kappa(W, X)=\kappa(Z, X)=\kappa[D] \geqq 0 .
$$

In the case ii-a), we have $\beta_{1}=\beta_{2}=3$ and so $x=y=1 / 3$ and $z=1$. Thus $W=K_{X}-\Gamma ; \kappa(W, X)=\kappa(X)=-\infty$. This implies $\kappa[D]=\kappa(W, X)=-\infty$, a contradiction.

In case ii-b), we have $z=2 / 5, x=2 / 15, y=1 / 10$. Hence

$$
W=K_{x}+\left(D_{1}+2 D_{2}\right) / 5-2 / 5 \cdot \Gamma \text {. }
$$

Contracting $\Gamma$ into a non-singular point $p$, we have a non-singular rational surface $Y$ and a birational morphism $\mu: X \rightarrow Y$. Let $\Delta_{i}=\mu\left(D_{i}\right)$ for $i=1$, 2. Denoting $K_{Y}+\left(\Delta_{1}+2 \Delta_{2}\right) / 5$ by $W_{1}$, we obtain

$$
W=\mu^{*}\left(W_{1}\right) \text {. }
$$

First, assume that $W_{1}$ is nef. Then since $\kappa\left(W_{1}, Y\right) \geqq 0$, it follows that $\left(W_{1}\right)^{2} \geqq 0$ and $\left(W_{1}\right)^{2}=W_{1} \cdot K_{Y}=\left(K_{Y}\right)^{2}+2 / 5$. Hence $\left(K_{Y}\right)^{2} \geqq 0$. We use the next

LEMMA 2. $\operatorname{dim}\left|-K_{Y}\right| \geqq\left(K_{Y}\right)^{2}$.

Proof. This follows from the Riemann-Roch theorem applied to a rational surface $Y$ (see Lemma 4 in [5]).

Hence $\left|-K_{Y}\right|$ is not empty and so $W_{1} \cdot\left(-K_{Y}\right) \geqq 0$. This induces $-\left(K_{Y}\right)^{2}-2 / 5 \geqq 0 ;$ thus $\left(K_{Y}\right)^{2} \leqq-2 / 5$, which contradicts $\left(K_{Y}\right)^{2} \geqq 0$. 
Therefore we can conclude that $W_{1}$ is not nef, i.e. there exists an irreducible curve $C$ such that $W_{1} \cdot C<0$.

Then $C^{2}<0$ and $C \cdot K_{Y}<0$. Hence $C$ is again an exceptional curve on $Y$. Letting $\varepsilon_{i}=\Delta_{i} \cdot C$ for $i=1,2$,

$$
W_{1} \cdot C=K_{Y} \cdot C+\left(\Delta_{1} \cdot C+2 \Delta_{2} \cdot C\right) / 5<0 .
$$

Hence we have

$$
\varepsilon_{1}+2 \varepsilon_{2}<5 \text {. }
$$

Since $(D, X)$ is relatively minimal, it follows that $\varepsilon_{1}+\varepsilon_{2} \geqq 2$. By $\kappa\left(W_{1}, Y\right) \geqq 0$, we can assume that $W_{1}$ is an effective $Q$-divisor. There exist non-negative integers $a, b, c$ and an effective $Q$-divisor $G$ such that $W_{1}=a \Delta_{1}+b \Delta_{2}+c C+G$, where $\operatorname{supp}(G)$ does not contain any irreducible components of $\Delta_{1}, \Delta_{2}$ and $C$. Then we have

$$
\begin{aligned}
& 0=W_{1} \cdot \Delta_{1} \geqq-2 a+b+c \varepsilon_{1}, \\
& 0=W_{1} \cdot \Delta_{2} \geqq a-3 b+c \varepsilon_{2}, \\
& 0=W_{1} \cdot C \geqq a \varepsilon_{1}+b \varepsilon_{2}-c,
\end{aligned}
$$

since $C \cdot \Delta_{\imath} \geqq 0$. Thus

$$
\begin{aligned}
& \varepsilon_{1}+2 \varepsilon_{2}<5, \\
& \varepsilon_{1}+\varepsilon_{2} \geqq 2, \\
& 2 a \geqq b+c \varepsilon_{1}, \\
& 3 b \geqq a+c \varepsilon_{2}, \\
& -1+\varepsilon_{1}+2 \varepsilon_{2} / 5 \geqq a \varepsilon_{1}+b \varepsilon_{2}-c .
\end{aligned}
$$

We claim that there exists no solution satisfying these inequalities. First, we consider the case in which $\varepsilon_{1}=2, \varepsilon_{2}=1$. Computing (i) +2 (iii), we get $-2 / 5 \geqq 3 b+2 a$. This is absurd, since $a, b \geqq 0$.

In the case when $\varepsilon_{1}=1$ and $\varepsilon_{2}=1$, computing (i) +(ii) $+2($ iii), we get $-4 / 5 \geqq a$. This contradicts the non-negativity of $a$.

Also in the case when $\varepsilon_{1} \geqq 2$ and $\varepsilon_{2}=0$, we can derive a contradiction by a similar argument. Therefore, the case ii-b) cannot occur.

We consider the case ii-c). We have $\beta_{1}=3, \beta_{2}=5, x=1 / 21, y=1 / 35$ and $z=1 / 7$. Then

$$
W=K+2\left(D_{1}+2 D_{2}\right) / 7-1 / 7 \cdot \Gamma .
$$

Contracting $\Gamma$ into a non-singular point $p$, we have a complete rational surface $Y$ and a birational morphism $\lambda: X \rightarrow Y$. Letting $K^{\prime}=K_{Y}, D_{1}^{\prime}=\lambda\left(D_{1}\right)$, 
$D_{2}^{\prime}=\lambda\left(D_{2}\right)$, and $W^{\prime}=K^{\prime}+2\left(D_{1}^{\prime}+2 D_{2}^{\prime}\right) / 7$, we have $\left(D_{1}^{\prime}\right)^{2}=-2, \quad\left(D_{2}^{\prime}\right)^{2}=-4$, $D_{1}^{\prime} \cdot D_{2}^{\prime}=1, W^{\prime} \cdot D_{1}^{\prime}=W^{\prime} \cdot D_{2}^{\prime}=0$. Further, we have $W=\lambda^{*}\left(W^{\prime}\right)$.

First assume that $W^{\prime}$ is nef. Then $\left(W^{\prime}\right)^{2} \geqq 0$ and $\left(W^{\prime}\right)^{2}=W^{\prime} \cdot K^{\prime}=$ $\left(K^{\prime}\right)^{2}+8 / 7$. Thus $\left(K^{\prime}\right)^{2} \geqq-1$ and

$$
\left(W^{\prime}\right)^{2}>0
$$

because $\left(K^{\prime}\right)^{2}$ is an integer.

If $\left(K^{\prime}\right)^{2} \geqq 0$, then from Lemma 2 applied to $K^{\prime}$, it follows that

$$
\operatorname{dim}\left|-K^{\prime}\right| \geqq\left(K^{\prime}\right)^{2} \geqq 0 \text {. }
$$

Thus $W^{\prime} \cdot\left(-K^{\prime}\right) \geqq 0$ since $W^{\prime}$ is nef. From this we have $\left(W^{\prime}\right)^{2}=W^{\prime} \cdot K^{\prime} \leqq 0$. But this contradicts $(*)$.

When $\left(K^{\prime}\right)^{2}=-1$, calculating $l\left(-K^{\prime}-D_{2}^{\prime}\right)$ by the Riemann-Roch theorem, we have

$$
\begin{gathered}
l\left(-K^{\prime}-D_{2}^{\prime}\right)+l\left(2 K^{\prime}+D_{2}^{\prime}\right) \geqq K^{\prime} \cdot\left(K^{\prime}+D_{2}^{\prime}\right) \\
=-1+K^{\prime} \cdot D_{2}^{\prime}=1 .
\end{gathered}
$$

If $l\left(2 K^{\prime}+D_{2}^{\prime}\right)=0$, then $\left|-K^{\prime}-D_{2}^{\prime}\right| \neq \varnothing$. Thus, $W^{\prime} \cdot\left(-K^{\prime}-D_{2}^{\prime}\right) \geqq 0$, since $W^{\prime}$ is nef. But $W^{\prime} \cdot\left(-K^{\prime}-D_{2}^{\prime}\right)=-W^{\prime} \cdot K^{\prime}=-1 / 7$, which contradicts the above. Therefore, we have $l\left(2 K^{\prime}+D_{2}^{\prime}\right)>0$. Since $\left(K^{\prime}\right)^{2}=-1$ and $\left(D_{2}^{\prime}\right)^{2}=-4$, by Lemma 7 in [5], we conclude that the pair $\left(D_{2}^{\prime}, Y\right)$ is relatively minimal and $\kappa\left[D_{2}^{\prime}\right]=0$ or 1 .

By the proof of Proposition 2 in [5], we have an exceptional curve $E$ such that $m K^{\prime}+D_{2}^{\prime} \sim(m-2) E$ for some $m>1$. (Note that the statement (ii) in Proposition 2 of [5] has some misprint; $K+D / 2 \sim(1 / m)(D+2 E)$ should be replaced by $K+D / 2 \sim((m-2) /(2 m))(D+2 E))$. Hence, taking the intersection number with $D_{1}^{\prime}$, we have

$$
1=(m-2) E \cdot D_{1}^{\prime} .
$$

Therefore, $m=3$ and $E \cdot D_{1}^{\prime}=1$. Moreover,

$$
D_{2}^{\prime} \cdot E=D_{2}^{\prime} \cdot\left(3 K^{\prime}+D_{2}^{\prime}\right)=-2+2 K^{\prime} \cdot D_{2}^{\prime}=2 .
$$

Contracting $E$ to a non-singular point, we have a complete nonsingular surface $V$, which is a rational elliptic surface with only one triple fiber.

The images of $D_{1}^{\prime}$ and $D_{2}^{\prime}$ are denoted by $C_{1}$ and $C_{2}$ respectively. $C_{1}$ is an exceptional curve and $C_{2}$ is a singular rational curve with one double point. Further,

$$
C_{1} \cdot C_{2}=3, \quad 3 K_{V}+C_{2} \sim 0, \quad\left(K_{V}\right)^{2}=0 .
$$


First, contract $C_{1}$ into a non-singular point and then contract all exceptional curves so that one can obtain a relatively minimal surface $F$. $F$ is $P^{2}$ or $\Sigma_{0}=P^{1} \times P^{1}$ or a Hirzebruch surface $\Sigma_{m}$ where $m \geqq 2$.

If $F$ is $\Sigma_{m}(m \geqq 2)$, then there exists a unique non-singular rational curve $\Delta_{\infty}$ such that $\left(\Delta_{\infty}\right)^{2}=-m$. The proper inverse image of $\Delta_{\infty}$ on $V$ is denoted by $\Gamma$. Then $\Gamma^{2} \leqq\left(\Delta_{\infty}\right)^{2}$. Let $k=-\Gamma^{2} \geqq m$.

For $F=\Sigma_{m}(m \geqq 0)$, we have $3 K_{V} \cdot \Gamma+C_{2} \cdot \Gamma=0$, and $K_{V} \cdot \Gamma=k-2$. Hence, $C_{2} \cdot \Gamma=3(2-k) \geqq 0$. Thus $m \leqq k \leqq 2$. Hence $m=2$ or 0 .

Further, note that if $m=2$ then $k=m$. This implies that the centers of blowing ups to obtain $V$ from $\Sigma_{m}$ do not lie on $\Delta_{\infty}$. Note the next easy lemma:

LEMMA 3. Let $\lambda: W \rightarrow \Sigma_{m}$ be a birational morphism which is not an isomorphism.

1) If $m=0$, then there exists a birational morphism from $W$ onto $\boldsymbol{P}^{2}$.

2) If $m>1$ and $\lambda^{-1}$ is regular on a neighborhood of $\Delta_{\infty}$, then there exists a birational morphism from $\Sigma_{m}$ onto $\Sigma_{m-1}$ such that the composed birational map: $W \rightarrow \Sigma_{m-1}$ is regular.

3) Under the same condition as in 2), if $m=2$, then there exists a birational morphism from $W$ onto $\boldsymbol{P}^{2}$.

Proof. 1) Since $\lambda$ is not the isomorphism, there exists a point $q$ at which $\lambda^{-1}$ is not regular, Take two lines $L=\{x\} \times P^{1}$ and $M=P^{1} \times\{y\}$ where $(x, y)$ denotes the coordinates of $q$. Blowing up $q$, we have a birational morphism: $W \rightarrow \Sigma_{1}$ and the strict transform $L^{\prime}$ and $M^{\prime}$ of $L$ and $M$ are exceptional curves on $\Sigma_{1}$. Blowing down these, we have $P^{2}$ and a birational morphism: $W \rightarrow \boldsymbol{P}^{2}$.

The proof of 2) is easy. 3) follows from 2).

By this lemma, we can assume $F$ to be $P^{2}$. The image of $C_{2}$ is denoted by $L$. Thus letting $H$ be a line on $P^{2}$ and letting $d$ be the degree of $L$, we have $L \sim d H$ and

$$
C_{2}+3 K_{V}=\mu^{*}((d-9) H)-\sum_{j=1}^{\dot{1}}\left(\nu_{j}-3\right) E_{j}
$$

where the $E_{j}$ are the exceptional curves arising from the singular points of multiplicities $\nu_{j}$.

Therefore $d=9$, and $\nu_{1}=\nu_{2}=\cdots=\nu_{9}=3$. Further, recalling that the multiplicity of the singular point of $C_{2}$ is 2 , we add $\nu_{10}=2$. Note that the tenth singular point is one of the infinitely near singular points of the plane curve $L$.

Moreover, if $(D, X)$ is obtained in the above way, we can compute 
the bigenus of $(D, X)$ and get $P_{2}[D]=1$. Indeed, since $\left(2 K_{X}+2 D\right) \cdot D_{i} \leqq-1$ for $i=1,2$, it follows that

$$
P_{2}[D]=l\left(2 K_{X}+D\right)
$$

Clearly,

$$
2 K_{X}+D=\lambda^{*}\left(2 K^{\prime}+D^{\prime}\right) \text { and }\left(2 K^{\prime}+D^{\prime}\right) \cdot D_{1}^{\prime}<0 \text {. }
$$

Hence,

$$
l\left(2 K_{x}+D\right)=l\left(2 K^{\prime}+D_{2}^{\prime}\right) .
$$

Since $V$ is an elliptic rational surface with one triple fiber, $D_{2}^{\prime}$ is a fiber and we have an effective curve $F_{1}$ such that $F_{1} \sim-K^{\prime}$ and $D_{2}^{\prime} \sim 3 F_{1}$. Hence

$$
2 K^{\prime}+D_{2}^{\prime} \sim F_{1} ; \text { thus } P_{2}[D]=1 \text {. }
$$

The pair $(D, X)$ with this property will be referred to as the pair of type (\$).

In the case when $W^{\prime}$ is not a nef $Q$-divisor, we can derive a contradiction by the same argument as in the previous case.

Therefore, except for the case (\$), we conclude that

$$
Z=K_{X}+\left(1-2 / \beta_{1}\right) D_{1}+\left(1-2 / \beta_{2}\right) D_{2}
$$

is the nef part of $K_{x}+D$.

Assume $Z^{2}>0$. By the vanishing theorem,

$$
H^{1}\left(X, \mathcal{O}\left(2 K_{X}+D_{1}+D_{2}\right)\right)=H^{1}\left(X, \mathcal{O}\left(-\left(K_{X}+D_{1}+D_{2}\right)\right)\right)=H^{1}(\operatorname{INT}(-Z))=0 \text {. }
$$

Hence, by the Riemann-Roch theorem,

$$
l\left(2 K_{X}+D\right)=K_{X} \cdot\left(K_{X}+D_{1}+D_{2}\right)-1=\left(K_{X}\right)^{2}+\beta_{1}+\beta_{2}-5 .
$$

From $Z^{2}>0$ it follows that

$$
Z^{2}=\left(K_{X}\right)^{2}+\beta_{1}+\beta_{2}-8+4\left(1 / \beta_{1}+1 / \beta_{2}\right)>0 .
$$

Hence,

$$
\left(K_{X}\right)^{2}+\beta_{1}+\beta_{2}-5>3-4\left(1 / \beta_{1}+1 / \beta_{2}\right) .
$$

On the other hand, we have

$$
4-4\left(1 / \beta_{1}+1 / \beta_{2}\right)>1 \quad \text { whenever } 3 \leqq \beta_{1} \leqq \beta_{2} .
$$

Accordingly, we have the following result. 
Proposition 2. Suppose that $D$ consists of two rational curves. If $\kappa[D] \geqq 0$ and $\beta_{1}>2$, then the nef part of $K_{X}+D$ is obtained as follows:

In case $(D, X)$ is of type (\$), $\lambda^{*}\left(K^{\prime}+\left(2 D_{1}^{\prime}+4 D_{2}^{\prime}\right) / 7\right)$ is the nef part. In this case, $P_{2}[D]=1$ and $\kappa[D]=2$.

In the other cases, $K_{X}+\left(1-2 / \beta_{1}\right) D_{1}+\left(1-2 / \beta_{2}\right) D_{2}$ is the nef part of $K_{X}+D_{1}+D_{2}$. Further, if $\kappa[D]=2$, then $P_{2}[D]=\left(K_{X}\right)^{2}+\beta_{1}+\beta_{2}-5 \geqq 1$.

REMARK. The case $\beta_{1}=3, \beta_{2}=3$ does not occur. Actually, otherwise we have $\left(K_{X}\right)^{2}+6+2 \cdot 4 / 3>8$. Then $\left(K_{X}\right)^{2}>-2 / 3$; hence $\left(K_{X}\right)^{2} \geqq 0$, which implies $\left|-K_{X}\right| \neq \varnothing$ by Lemma 2 . Since $Z$ is nef, $-K_{X} \cdot Z \geqq 0$. Hence $-Z \cdot Z=-K_{X} \cdot Z \geqq 0$ and so $Z^{2} \leqq 0$. But this cannot happen.

It seems that there exist no relatively minimal pairs with $\beta_{1}=3$, $\beta_{2}=4$ or $\beta_{2}=5$. Such a problem will be discussed.

§4. We study the case $\kappa[D]=0$ or 1 , where $D$ consists of two rational curves. By the last proposition, letting

$$
Z=K_{X}+\left(1-2 / \beta_{1}\right) D_{1}+\left(1-2 / \beta_{2}\right) D_{2},
$$

we have $Z^{2}=0$. We assume $3 \leqq \beta_{1} \leqq \beta_{2}$. Since

$$
Z^{2}=\left(K_{X}\right)^{2}+\beta_{1}+\beta_{2}+4\left(1 / \beta_{1}+1 / \beta_{2}\right)-8,
$$

we obtain the following cases;

1) $\beta_{1}=4, \beta_{2}=4$,

2) $\beta_{1}=8, \beta_{2}=8$,

3) $\beta_{1}=6, \beta_{2}=12$,

4) $\beta_{1}=3, \beta_{2}=6$,

5) $\beta_{1}=5, \beta_{2}=20$.

Actually, if $\beta_{1}=\beta_{2}$, then it is 4 or 8 . In the other cases, we have $\beta_{1} \leqq 6$. Clearly, $\beta_{1}=6$ or 5 or 3 and then we have only five cases listed as above. But we shall show that the cases 2) through 5) do not occur.

Suppose that the case 4 ) occurs. Then $Z=K_{X}+D_{1} / 3+2 / 3 \cdot D_{2}$; hence

$$
0=Z \cdot K_{X}=\left(K_{X}\right)^{2}+D_{1} \cdot K_{X} / 3+2 D_{2} \cdot K_{X} / 3 \text {. }
$$

Thus, $\left(K_{X}\right)^{2}=-3$.

Case $1 . \quad \kappa[D]=0$. Then $3 m Z \sim 0$ for some $m>0$. Since $X$ is simply connected, it follows that

$$
3 Z=3 K_{X}+D_{1}+2 D_{2} \sim 0 .
$$

Since $\left(D_{1}\right)^{2}=-3$, we have $\kappa\left[D_{1}\right]=-\infty$ by Lemma 1 . Moreover $\left(K_{X}\right)^{2}=-3$ implies that $\left(D_{1}, X\right)$ cannot be relatively minimal. Hence there exists an exceptional curve $E$ on $X$ such that $D_{1} \cdot E \leqq 1$. If $D_{1} \cdot E=0$, then 


$$
0=Z \cdot E=3 K_{X} \cdot E+D_{1} \cdot E+2 D_{2} \cdot E=-3+2 D_{2} \cdot E,
$$

which is a contradiction. Thus $D_{1} \cdot E=1$ and so $D_{2} \cdot E=1$. Contracting $E$ into a non-singular point, we have a non-singular rational surface $Y$ and the images $D_{1}^{\prime}$ and $D_{2}^{\prime}$ of $D_{1}$ and $D_{2}$. Then

$$
\begin{aligned}
& 3 K_{Y}+D_{1}^{\prime}+2 D_{2}^{\prime} \sim 0 \quad \text { and } \\
& \left(D_{1}^{\prime}\right)^{2}=-2, \quad\left(D_{2}^{\prime}\right)^{2}=-5, \quad\left(K_{Y}\right)^{2}=-2 .
\end{aligned}
$$

We repeat this process once more. We have a non-singular rational surface $Z$ and non-singular rational curves with $\left(D_{1}^{\prime \prime}\right)^{2}=-1$ and $\left(D_{2}^{\prime \prime}\right)^{2}=-4$. Moreover, $3 K_{Z}+D_{1}^{\prime \prime}+2 D_{2}^{\prime \prime} \sim 0$. Now $D_{1}^{\prime \prime}$ is an exceptional curve, we have a non-singular rational surface $W$ and the image $H$ of $D_{2}^{\prime \prime}$ satisfies that

$$
3 K_{W}+2 H \sim 0 \text {. }
$$

$W$ has an exceptional curve $L$ and then $3 K_{1} \cdot L+2 H \cdot L=0$, which is a contradiction.

Case 2. $\kappa[D]=1$.

Claim. There exists an exceptional curve $E$ such that $Z \cdot E=0$.

Actually, by a theorem of Kawamata [6], $Z$ is semiample, in other words, one has a positive number $m$ such that $|m Z|$ has no base points. The rational map defined by $m\left(K_{X}+D\right)$ for $m \gg 0$ is a morphism $f$ onto a projective line $B$. $f$ coincides with the morphism defined by $m Z$ for $m \gg 0$. Hence, denoting by $C_{u}$ a general fiber of $f$, we have $a>0$ such that $Z \sim a C_{u}$. Then $Z \cdot D_{i}=0$ induces $C_{u} \cdot D_{i}=0$. On the other hand,

$$
0=Z \cdot C_{u}=K_{X} \cdot C_{u}+D_{1} \cdot C_{u} / 3+D_{2} \cdot 2 C_{u} / 3
$$

From this we derive $0=K_{X} \cdot C_{u}$ and hence $\pi\left(C_{u}\right)=1$, where $\pi(C)$ denotes the virtual genus of $C . C_{u}$ is an elliptic curve and thus $f: X \rightarrow B$ is an elliptic surface. However, since $\left(K_{X}\right)^{2}<0$, there exists an exceptional curve $E$ in a fiber. Therefore, $E \cdot Z=a\left(E \cdot C_{u}\right)=0$; hence

$$
3=E \cdot D_{1}+2 E \cdot D_{2} \text {. }
$$

If $E \cdot D_{2}=0$ then $E \cdot D_{1}=3$. But contracting $E$, we have a birational morphism $\mu: X \rightarrow Y$ and the image $D_{1}^{\prime}$ of $D_{1}$ satisfies that $\left(D_{1}^{\prime}\right)^{2}=6$, which implies that $D_{1}^{\prime}$ cannot be contained in a fiber, a contradiction. Hence, we obtain $E \cdot D_{2}>0$ and then $E \cdot D_{1}=E \cdot D_{2}=1$. Note that in this case, both $D_{1}$ and $D_{2}$ are contained in the same fiber. Contracting $E$, we have a birational morphism $\mu: X \rightarrow Y$ and the images $D_{i}^{\prime}$ of $D_{i}$ for $i=1,2$ satisfy that $\left(D_{1}^{\prime}\right)^{2}=-2$ and $\left(D_{2}^{\prime}\right)^{2}=-5$. Letting $Z^{\prime}=K_{Y}+\left(D_{1}^{\prime}+2 D_{2}^{\prime}\right) / 3$, we have $Z=\mu^{*}\left(Z^{\prime}\right)$. And $\left(Z^{\prime}\right)^{2}=0,\left(K_{Y}\right)^{2}=-2$. Repeating this process twice more, 
we have the images $D_{1}^{(3)}$ and $D_{2}^{(3)}$ which satisfy $\left(D_{1}^{(3)}\right)^{2}=0$ and $\left(D_{2}^{(3)}\right)^{2}=-3$. But these cannot be contained in a singular fiber.

In the case 5), we have

$$
10 Z \sim 10 K_{X}+3\left(2 D_{1}+3 D_{2}\right) .
$$

By a similar argument as in the former case, we have an exceptional curve $E$ such that $Z \cdot E=0$. Then

$$
0=10 Z \cdot E=10 K_{X} \cdot E+3\left(2 D_{1} \cdot E+3 D_{2} \cdot E\right) \text {. }
$$

But this is impossible.

By the same reasoning, also in the cases 2 ) and 3), we can derive contradictions. However, the case 1$)$ survives.

In this case, we have $\beta_{1}=\beta_{2}=4$ and $\left(K_{X}\right)^{2}=-2$.

Case $\kappa[D]=0$. We have $2 \nu Z \sim 0$ for some $\nu>0$; hence $2 K_{X}+D \sim 0$ since $X$ is simply connected. By Lemma 3, there exists a birational morphism $\mu: X \rightarrow P^{2}$ and we have a plane curve $C=\mu(D)$. Since $2 K_{X}+D \sim 0, C$ is a curve of degree 6 with only double points.

We claim that $C$ is reducible. Actually, if $C$ is irreducible, we may assume that $\mu\left(D_{1}\right)=C$ and $\mu\left(D_{2}\right)$ is a point. Then $\kappa\left[D_{1}\right]=\kappa[C]=0$; hence $P_{2}\left[D_{1}\right]=1$. However, $2 K_{X}+D_{1} \sim-D_{2}$ implies that $P_{2}\left[D_{1}\right]=0$, which contradicts the above fact. Thus we have two curves $C_{i}=\mu\left(D_{i}\right)$ for $i=1,2$.

Let $a=\operatorname{deg}\left(C_{1}\right), b=\operatorname{deg}\left(C_{2}\right)$. Since $a+b=6$, we have the three cases

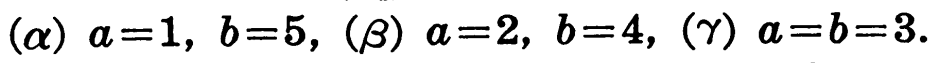

Case $(\alpha)$. Take three points $P, Q, R$ from the singular points of $C_{2}$, which are not colinear. Perform the Cremona transformation $\psi$ with centers $P, Q, R$. The transforms $C_{1}^{\prime}, C_{2}^{\prime}$ of $C_{1}$ and $C_{2}$ by $\psi$ satisfy the condition of the case $(\beta)$.

Case $(\beta)$. Take a point $P$ from the intersection of $C_{1}$ and $C_{2}$ and take two points $Q, R$ from the set of singular points of $C_{2}$ such that these are not colinear. Again perform the Cremona transformation with centers $P, Q, R$. Then we arrive at the case $(\gamma)$.

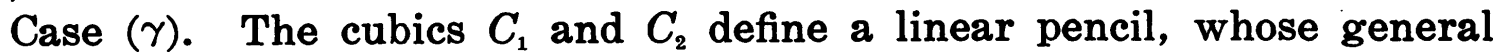
member is an elliptic curve. This implies that there exist exceptional curves $E_{1}, E_{2}$ on $X$ such that $D_{1} \cdot E_{1}=D_{2} \cdot E_{1}=D_{1} \cdot E_{2}=D_{2} \cdot E_{2}=1$.

By contracting these exceptional curves, we have a birational morphism $\mu: X \rightarrow Y$ such that $Y$ is an elliptic rational surface and the image of $D_{1}+D_{2}$ is a fiber of a fiber space $f: Y \rightarrow B$ of the elliptic surface $Y$.

Case $\kappa[D]=1$. The $Q$-divisor $Z$ defines a morphism, which is denoted by $h: X \rightarrow B_{1}$. For a general fiber $C_{k}$ of $h$, we have

$$
0=2 Z \cdot C_{u}=2 K_{x} \cdot C_{u}+D_{1} \cdot C_{u}+D_{2} \cdot C_{u},
$$


and thus we have $K_{X} \cdot C_{u}=0$ or -2 .

If $K_{X} \cdot C_{u}=0$, then $C_{u}$ is an elliptic curve and $D_{1} \cdot C_{u}=D_{2} \cdot C_{u}=0$. We let $p_{j}=h\left(D_{j}\right)$ for $j=1,2$.

If $p_{1} \neq p_{2}$, then take exceptional curves $E_{j}$ such that $h\left(E_{j}\right)=p_{j}$ for $j=1,2$ and that $D_{1} \cdot E_{1}=D_{2} \cdot E_{2}=2, D_{1} \cdot E_{2}=D_{2} \cdot E_{1}=0$. Contracting these $E_{j}$, we have a birational morphism $\mu: X \rightarrow Y$ and the images $D_{j}^{\prime}$ of $D_{j}$ are singular fibers of the fiber space of the elliptic surface $Y$. By a canonical bundle formula of $Y$, we have $\nu K_{Y}+D_{1}^{\prime}+D_{2}^{\prime} \sim 0$ for some $\nu \geqq 3$. From this we readily infer $P_{2}\left[D_{1}+D_{2}\right] \geqq 1$.

If $p_{1}=p_{2}$, then there exist exceptional curves $E_{1}$ and $E_{2}$ such that $D_{1} \cdot E_{1}=D_{2} \cdot E_{1}=D_{1} \cdot E_{2}=D_{2} \cdot E_{2}=1$. Contracting these $E_{1}$ and $E_{2}$, we have a reducible curve $D_{1}^{\prime}+D_{2}^{\prime}$ which is a singular fiber consisting of nonsingular rational curves with intersection number 2. By a canonical bundle formula, we have $\nu$ such that $\nu K_{Y}+D_{1}^{\prime}+D_{2}^{\prime} \sim 0$, where $\nu \geqq 3$. Hence, $P_{2}\left[D_{1}+D_{2}\right] \geqq 1$.

Finally we shall show that $K_{X} \cdot C_{u}=-2$ does not occur. In such a case, we have $\left(D_{1}+D_{2}\right) \cdot C_{u}=4$, and $C_{u}$ is a rational curve. Thus the following three cases may occur: (i) $C_{u} \cdot D_{1}=C_{u} \cdot D_{2}=2$, (ii) $C_{u} \cdot D_{1}=3$, $C_{u} \cdot D_{2}=1$, (iii) $C_{u} \cdot D_{1}=4, C_{u} \cdot D_{2}=0$. Blowing down an exceptional curve $E$ contained in a fiber of $h: X \rightarrow B_{1}$, we have a birational morphism $\mu_{1}: X \rightarrow X_{1}$ and $0=2 Z \cdot E=-2+\left(D_{1}+D_{2}\right) \cdot E$. Hence the curve $D_{1}^{\prime}+D_{2}^{\prime}$ obtained from $D_{1}+D_{2}$ has a double point and

$$
2 K_{X}+D_{1}+D_{2}=\mu_{1}^{*}\left(2 K_{X_{1}}+D_{1}^{\prime}+D_{2}^{\prime}\right) .
$$

Repeating this process, we finally obtain a Hirzebruch surface $Y=\Sigma_{b}$, $b \geqq 0$.

Let $\mu: X \rightarrow Y$ be the composition of the blowing downs, and let $C_{t}=$ $\mu\left(D_{i}\right)$. Then the curve $C=C_{1}+C_{2}$ has only double points. By adjunction formula, $\left(C_{i}\right)^{2}+K_{Y} \cdot C_{i}=2 \pi_{i}-2, \pi_{i}$ being the virtual genus of $C_{i}$, for $i=$ 1, 2. Since $C$ has only double points and each $C_{i}$ is rational,

$$
\left(C_{i}\right)^{2}-4 \pi_{i}-C_{1} \cdot C_{2}=\left(D_{i}\right)^{2}=-4 \text {. }
$$

Recall that the Picard group $\operatorname{Pic}(Y)$ is generated by a fiber $F$ and a section $\Delta_{\infty}$ with $\left(\Delta_{\infty}\right)^{2}=-b$. In case (i), since $C_{i} \cdot F=2$,

$$
C_{i} \sim 2 \Delta_{\infty}+k_{i} F \quad \text { for some } k_{i}>0 \text {. }
$$

Then

$$
\begin{aligned}
& K_{Y} \sim-2 \Delta_{\infty}-(2+b) \cdot F, \\
& C_{1} \cdot C_{2}=2\left(k_{1}+k_{2}\right)-4 b,
\end{aligned}
$$




$$
\begin{aligned}
& \pi_{i}=k_{i}-b-1, \\
& \left(C_{i}\right)^{2}=4\left(k_{i}-b\right), \\
& 2 K_{Y}+C_{1}+C_{2} \sim\left(k_{1}+k_{2}-2 b-4\right) F .
\end{aligned}
$$

From $\left(C_{i}\right)^{2}-4 \pi_{i}-C_{1} \cdot C_{2}=-4$, it follows that

$$
2 b=k_{1}+k_{2}-4 \text {. }
$$

Thus $2 K_{Y}+C_{1}+C_{2} \sim\left(k_{1}+k_{2}-2 b-4\right) F=0$. This implies that $Z \sim 0$ on $X$, which contradicts the hypothesis that $\kappa[D]=\kappa(Z, X)=1$. Similarly we can rule out the cases (ii) and (iii).

As a consequence of the above argument, we obtain the following result.

Proposition 3. The relatively minimal pairs $(D, X)$ with $\kappa[D]=0$ or 1 are obtained from a rational elliptic surface $f: V \rightarrow B$ by blowing up as follows:

Case a). There exists a singular fiber consisting of two irreducible rational curves $C_{1}$ and $C_{2}$. By performing blowing ups to separate $C_{1}$ and $C_{2}$, we have a surface $X$ and a required reducible curve $D$.

Case b). There exist two singular rational irreducible fibers $C_{1}$ and $C_{2}$. Then blowing up these singular points, we have $X$ and the proper transforms $D_{1}$ and $D_{2}$. Then $D=D_{1}+D_{2}$.

Case c). $(D, X)$ is obtained from $\left(D^{\prime}, Y\right)$ with a singular irreducible fiber $D^{\prime}$ such that there exists a birational morphism $\mu: X \rightarrow Y$ and that $D=\mu^{-1}\left(D^{\prime}\right)+\Gamma$, where $\Gamma$ is a non-singular rational curve with $\Gamma^{2}=-2$.

COROLLARY. Under the above condition, if $\kappa[D]=0$ or 1 , then $P_{2}[D] \geqq 1$.

THEOREM 1. Let $C$ be a curve of two irreducible components on a projective plane. Then $C$ is transformed into a union of two lines by a Cremona transformation if and only if $P_{2}[C]=0$.

Proof. By Theorem in [4], it suffices to show that $\kappa[D]=-\infty$ is derived from $P_{2}[D]=0$. Actually, $P_{2}[D]=0$ induces $P_{1}[D]=0$, which implies that each component is a rational curve. Thus applying Proposition 2, from $P_{2}[D]=0$ we conclude that $\kappa[D]<2$. Further, by Corollary to Proposition 3 , if $\kappa[D]=0$ or 1 then $P_{2}[D] \geqq 1$. Hence $\kappa[D]=-\infty$ is derived from $P_{2}[D]=0$.

$\S 5$. We shall study non-rational case, in other words, the case in which $D_{1}$ is not rational. Let $g_{1}=\pi\left(D_{1}\right)$ and $g_{2}=\pi\left(D_{2}\right)$. We suppose that 
$g_{1}>0$. Further, as before, we assume that $\left(D_{1}+D_{2}, X\right)$ is relatively minimal. Note that in this case, $D_{2}$ is assumed to be not exceptional. Then it is easy to verify the following proposition.

Proposition 4. Suppose that $g_{1}>0$.

1) If $g_{2}>0$, then $Z=K_{X}+D_{1}+D_{2}$ is nef.

2) If $g_{2}=0$, then $\beta=-\left(D_{2}\right)^{2}>1$, and letting $Z=K_{X}+D_{1}+(1-2 / \beta) D_{2}$, $Z$ is nef.

The proof is similar to that of Lemma 1 of [4].

REMARK. In the second case, we have

(1) $Z \cdot D_{2}=0$ and $Z \cdot D_{1}=2 g_{1}-2 \geqq 0$,

(2) $Z+2 / \beta \cdot D_{2}$ is the Zariski decomposition of $K_{X}+D_{1}+D_{2}$. Thus, by a theorem of Kawamata [6, 7], $Z$ is semiample.

The purpose of this section is to decide the type of $(D, X)$, when $\kappa[D]<2$. In this case, we have $Z^{2}=0$.

First, assume $g_{2}>0$. We shall prove that $\kappa[D]=1$. Otherwise, $\kappa[D]=0$ and then $Z$ is numerically equivalent to 0 . If $X$ is not a relatively minimal surface, there exists an exceptional curve $E$. Then $Z \cdot E=0$, and $-1=D_{1} \cdot E+D_{2} \cdot E$. This contradicts the minimality of the pair $(D, X)$. Thus $X$ turns out to be a projective plane or a Hirzebruch surface. Clearly, we have no such a curve $D=D_{1}+D_{2}$ on $X$.

Now $\kappa[D]=1$ is proved and $Z$ defines a fiber space $f: X \rightarrow B$, whose general fiber is denoted again by $C_{u}$.

We note that there is no exceptional curve in fibers of $f: X \rightarrow B$. Indeed, an exceptional curve $E$ in a fiber satisfies

$$
-1=K_{X} \cdot E=-D_{1} \cdot E-D_{2} \cdot E .
$$

This contradicts the relative minimality of $\left(D_{1}+D_{2}, X\right)$.

From $Z \cdot C_{u}=0$, we have $K_{X} \cdot C_{u}=-D_{1} \cdot C_{u}-D_{2} \cdot C_{u} \leqq 0$. Hence we have two cases.

Case $K_{X} \cdot C_{u}=0 . \quad f: X \rightarrow B$ is an elliptic surface and $D_{1} \cdot C_{u}=D_{2} \cdot C_{u}=0$. Hence, both $D_{1}$ and $D_{2}$ are elliptic curves which are fibers.

Case $K_{X} \cdot C_{u}=-2 . \quad C_{u}$ is a rational curve and then $X$ is a $\boldsymbol{P}^{1}$-bundle over $\boldsymbol{P}^{1}$, since there are no exceptional curves in fibers. Clearly, on such a surface $X, D_{1}+D_{2}$ cannot lie.

Now suppose that $g_{2}=0$. Then $Z=K_{X}+D_{1}+(1-2 / \beta) D_{2}$ is nef. In this case, if $\beta=2$, then $Z=K_{X}+D_{1}$. As in the proof of Proposition 1 , the study of $\left(D_{1}+D_{2}, X\right)$ is reduced to that of $\left(D_{1}, X\right)$. Hence, supposing that 
$\beta>2$, we shall study the pairs $(D, X)$ with $\kappa[D]=0$ or 1 . We claim that $\kappa[D]=0$ is impossible. Actually, if $\kappa[D]=0$, then $\beta Z \sim 0 . g_{1}$ being positive, $\left|K_{1}+D_{1}\right| \neq \varnothing$ and take $\Delta$ from this. Then $0 \sim \beta Z \sim \beta \Delta+(\beta-2) D_{2}$, which is impossible. Hence we have $\kappa[D]=1$. Then $Z^{2}=0$ and by computation,

$$
Z^{2}=2 K_{X} \cdot D_{1}+\left(D_{1}\right)^{2}+\left(K_{X}\right)^{2}+(1-2 / \beta)(\beta-2) .
$$

Hence, $\beta-4+4 / \beta$ is an integer; thus $\beta=4$.

$Z$ defines a fiber space $f: X \rightarrow B$ with general fiber $C_{u}$. Since $Z$ is semiample, we have $Z \cdot C_{u}=0$. Then $\pi\left(C_{x}\right)=0$ or 1 .

Case $\pi\left(C_{u}\right)=1$. In this case, we have $K_{X} \cdot C_{u}=0$ and $D_{1} \cdot C_{u}=D_{2} \cdot C_{u}=0$. Since $g_{1}>0$ and $g_{2}=0, D_{1}$ turns out to be some fiber of $f$ and $D_{2}$ is a part of a fiber. From $Z \cdot K_{X}=\left(K_{X}\right)^{2}+D_{1} \cdot K_{X}+D_{2} \cdot K_{X} / 2=\left(K_{X}\right)^{2}+1$ and $Z \cdot K_{X}=0$, it follows that $\left(K_{X}\right)^{2}=-1$. Hence there exists an exceptional curve $E$ in a fiber of the elliptic surface $X$. Thus $Z \cdot E=0$ and $K_{X} \cdot E+$ $D_{1} \cdot E+D_{2} \cdot E / 2=0$. Then $D_{2} \cdot E=2$. After contracting $E$ into a non-singular point, we have a birational morphism $\mu: X \rightarrow Y$ and the proper image $D_{2}^{\prime}$, which is a rational curve with a double point.

Case $\pi\left(C_{u}\right)=0$. Then $K_{X} \cdot C_{u}=-2$ and $Z \cdot C_{u}=K_{X} \cdot C_{u}+D_{1} \cdot C_{u}+D_{2} \cdot C_{u} / 2=0$. We have the following three cases:

(1) $D_{1} \cdot C_{u}=2$ and $D_{2} \cdot C_{u}=0$. Since $D_{2}$ is a proper subset of a fiber, there exists an exceptional curve $E$ in some fiber. Then

$$
0=Z \cdot E=K_{X} \cdot E+D_{1} \cdot E+D_{2} \cdot E / 2 \text {. }
$$

Since $(D, X)$ is relatively minimal, we have

$$
D_{1} \cdot E=0 \text { and } D_{2} \cdot E=2 \text {. }
$$

$D_{2}$ and $E$ are parts of singular fibers. But we shall show that this is impossible.

Contracting $E$, we have a birational morphism $\mu: X \rightarrow Y$ and the images $D_{i}^{\prime}=\mu\left(D_{t}\right)$. Since $D_{2} \cdot E=2, D_{2}^{\prime}$ has a double point and $\left(D_{2}^{\prime}\right)^{2}=$ $-4+4=0$. Thus $D_{2}^{\prime}$ is a singular fiber of the fiber space $f^{\prime}: Y \rightarrow B$ obtained from $f: X \rightarrow B$. Further, $D_{1}^{\prime} \cdot D_{2}^{\prime}=0$ since $D_{1} \cdot E=0$ and $D_{1} \cdot D_{2}=0$. However, from $D_{1} \cdot C_{k}=2$ and $C_{k}^{\prime} \sim D_{2}^{\prime}$, it follows that $D_{1}^{\prime} \cdot D_{2}^{\prime}=2$, which contradicts the above. Hence, this case does not occur.

(2) $D_{1} \cdot C_{u}=1$ and $D_{2} \cdot C_{u}=2$. Then $D_{1}$ corresponds birationally to $B$, which is a rational curve. This contradicts $g_{1}>0$.

(3) $D_{1} \cdot C_{u}=0$ and $D_{2} \cdot C_{u}=4$. Then $D_{1}$ is a part of a fiber of the fiber space of rational curves. This implies that $D_{1}$ is a rational curve, which contradicts the hypothesis. 
Thus summarizing the above discussion, we obtain the following result:

Proposition 5. The relatively minimal pairs $(D, X)$ with nonrational $D_{1}$ and $\kappa\left[D_{1}+D_{2}\right]<2$ are as follows:

(1) If $g_{1}>0$ and $g_{2}>0$, then both $D_{1}$ and $D_{2}$ are non-singular fibers of a rational elliptic surface.

(2) If $g_{1}>0$ and $g_{2}=0$, then either there exists a birational morphism $h: X \rightarrow V$ such that $V$ is an elliptic surface with $\left(K_{V}\right)^{2}=0$ and the image of $D_{1}$ is a non-singular fiber and the image of $D_{2}$ is an irreducible singular fiber or $X$ is an elliptic surface with $\left(K_{X}\right)^{2}=0$ and $D_{1}$ is a fiber and $D_{2}$ is a part of the singular fiber.

Except for the last case, $\kappa\left[D_{1}+D_{2}\right]=1$.

REMARK. The last case in (2) corresponds to the case $\beta=2$.

\section{References}

[1] J. L. Coolidge, A Treaties on Algebraic Plane Curves, Oxford Univ. Press, 1928.

[2] D. Dicks, Birational geometry according to S. Iitaka, Univ. of Warwick, 1985.

[3] S. IITAKA, Basic structure of algebraic varieties, Algebraic Varieties and Analytic Varieties, Adv. Stud. Pure Math., 1 (1982), 303-316, Kinokuniya/North-Holland.

[4] S. IItaka, On a characterization of two lines on a projective plane, Proc. Algebraic Geometry, Lecture Notes in Math., 1016 (1983), 432-448, Springer.

[5] S. IttakA, On irreducible plane curves, Saitama Math. J., 1 (1983), 47-63.

[6] Y. Kawamata, On the classification of non-complete algebraic surfaces, Proc. Algebraic Geometry, Kopenhagen 1978, Lecture Notes in Math., 732 (1978), 215-332, Springer.

[7] M. MiYanishi, Non-complete Algebraic Surfaces, Lecture Notes in Math., 857 (1981), Springer.

Present Address:

Department of Mathematics, Gakushuin University

MejIRo, TOShIMA-KU, TOKYo 171 , JapaN 